\title{
Machine Vision Based Autonomous Green AgriBot
}

\author{
Gopireddy Sirisha, Parmar Harshdeepsinh, Konduri Subrahmanya Hemanth, \\ Sathiya Narayanan
}

\begin{abstract}
Automated agriculture bot offers effective solutions to modern agriculture. This paper explains the proposed multi-purpose automatic bot using machine vison-based navigation system powered by sun tracking solar panel with battery charger system with an overview on newly combined methodologies for the efficient improvement in production as well as in multiple agricultural operations like levelling, seed distribution and spraying, with effective power management system. This proposed idea will make the process sustainable and eco-friendly.
\end{abstract}

Keywords: Agricultural Bot, Row guidance system, Solar Power, Battery-management, Maximum Power Point Tracking, Internet of things.

\section{INTRODUCTION}

Robotics has transformed almost all the industrial sectors of our global economy and made notable contributions to various fields. However, it lags in efficiently improving the agricultural sector. Most of the farmers prefer conventional methods for agricultural practices as they are used to them. Advent of robotics into the agricultural sector has already resulted in remarkable changes in the way we harvest. And it has more to offer in other fields of agricultural operations to solve the two main problems, i.e., labor and time. Precision is an added advantage. Automation can solve these problems. Employment of proper navigation techniques results in the progress of future autonomous bots. Though machine vision helps to resolve navigation issues, navigation in amorphous and anonymous areas is still a fundamental trouble. This navigation challenge involves defining safe and efficient traversal paths to avoid obstacles and reach the target in the most effective way. Use of ranging sensors is a standard approach. Machine vision along with Global Positioning System (GPS) is a better method to tackle navigation problems. In this method the bot is self-localized, i.e. bot is aware of its position. A technique called Monte Carlo Localization (MCL) is used to estimate the location of the bot.

Revised Manuscript Received on December 16, 2019.

* Correspondence Author

Gopireddy Sirisha, School of Electronics Engineering (SENSE), Vellore Institute of Technology, Chennai (Tamil Nadu), India - 600127. Email: sirishareddy.grr@gmail.com

Parmar Harshdeepsinh*, School of Electronics Engineering (SENSE), Vellore Institute of Technology, Chennai (Tamil Nadu), India - 600127. Email: hdparmar1798@gmail.com

Konduri Subrahmanya Hemanth, School of Electronics Engineering (SENSE), Vellore Institute of Technology, Chennai (Tamil Nadu), India 600127. Email: hkounduri@gmail.com

Sathiya Narayanan, School of Electronics Engineering (SENSE), Vellore Institute of Technology, Chennai (Tamil Nadu), India - 600127. Email: sathiyanarayanan.s@vit.ac.in
Many vision and range sensors make MCL capable of locating the bot [1].

Though systems with solar are proved efficient with rechargeable batteries, their usage in robotics is not widespread as in the absence of sunlight the depleted battery doesn't get charged. An automated bot can be powered by a solar panel, which is designed to orient itself to the maximum intensity of light using solar tracking. The solar tracker will generate more power compared to stationary counterparts due to its exposure to maximum intensity light. This increase can be as much as 10 to $25 \%$ depending upon the farm size. And the battery management system with its dual monitor method controls and determines the capacity of both the charging and discharging batteries which helps to maintain the continuous availability of power to the bot [2].

\section{PROPOSED SYSTEM}

We propose a machine vision-based automated sun trackingsolar bot, as shown in Figure 1, with battery management system using Raspberry Pi 3 B connected to farmer through IoT, to help them reduce manual labor by performing different agricultural operations. The traversal path of the bot is defined by machine vision algorithm with the help of vision and ultrasonic sensors. At first one of the three operating modes needs to be selected and the end point needs to be specified, then the bot functions accordingly.

\section{A. Modes}

Levelling: A sheet of mild steel metal plate is arranged at the back of the bot with nuts and bolts for pitch (up \& down) motion of the plate. A leveler to the frame is fixed to level and close the sowed soil.

Seeds distribution: In forward motion the bot travels for few seconds and stops to dig the soil with the diggers placed on the center of the frame. These diggers are adjustable and are fixed by using nuts and bolts. A funnel is connected to these diggers through hoses. And the seeds are passed through this funnel-hose-digger passage and sowed.

Spraying (To control weeds): The complete flow is mentioned in Figure 1, while in straight linear motion the bot travels for few seconds and stops to capture an image using vision sensor attached in front of the bot. This image is analyzed by machine vision weed detection and classification algorithms on Raspberry Pi. If weeds are not detected, the bot moves forward in its path and continues to capture and analyze the images. If weeds 


\section{Machine Vision Based Autonomous Green AgriBot}

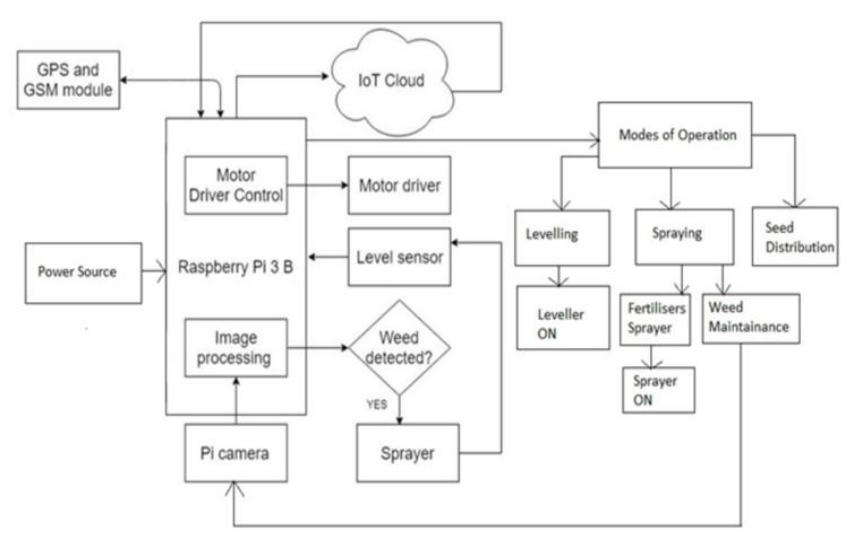

Fig. 1. Block diagram of the proposed system.

are detected, then sprayer will be switched on for some duration based on the classification and density of the weeds and is then switched off. After this the bot moves forward and repeats the process until the end point specified is reached.

Spraying (To spray fertilizer): It continuously switches the sprayer on along the path. It has sprayers on both the sides of the bot, they will be switched on alternatively based on the direction of the bot.

Along with this Raspberry Pi continuously monitors the liquid level in sprayer using level sensor. If it is empty, then the bot will stop and send an intimation message of present location coordinates to the farmer. This is done by using GPS and Global System for Mobile Communication (GSM) modules. If the farmer is willing to switch off the bot or specific functionalities of bot, he can do through cloud. But switching on should done manually as once completely switched off, it cannot stay connected. Alternatively, we can just switch off all other functionalities and keep the Raspberry Pi connected, but that is a wastage of energy. It is critical to get notified when any issues arise on the battery and battery management system side. As the battery management system functions continuously and monitors the battery performance it may malfunction due to external factors. If any unexpected situation arises then a notification will be sent to the cloud to notify the user.

\section{PHOTOVOLTAIC SYSTEM WITH SOLAR TRACKING}

Knowledge of Photovoltaic (PV) current-voltage (I-V) characteristics helps us to design a solar power array that operates closest to its maximum peak power point. A Photovoltaic module produces maximum

power, when solar radiation of $1000 \mathrm{~W} / \mathrm{m}^{2}$ metre falls on it. This graph in Figure 2 explains the I-V characteristics of a nor- mal $\mathrm{Si}$ Photovoltaic module placed in normal circumstances. Product of current and voltage gives the power produced. Obtain the power curve for voltages ranging from short-circuit to open-circuit conditions, given a level of radiation. At certain values of ' $I$ ' and ' $V$ ', the power ' $\mathrm{P}$ ' gets maximum, at 'Imp' and 'Vmp', defined as the "maximum power point (MPP)", the ideal operation point. It is obtained close to the bend in current-voltage curve.

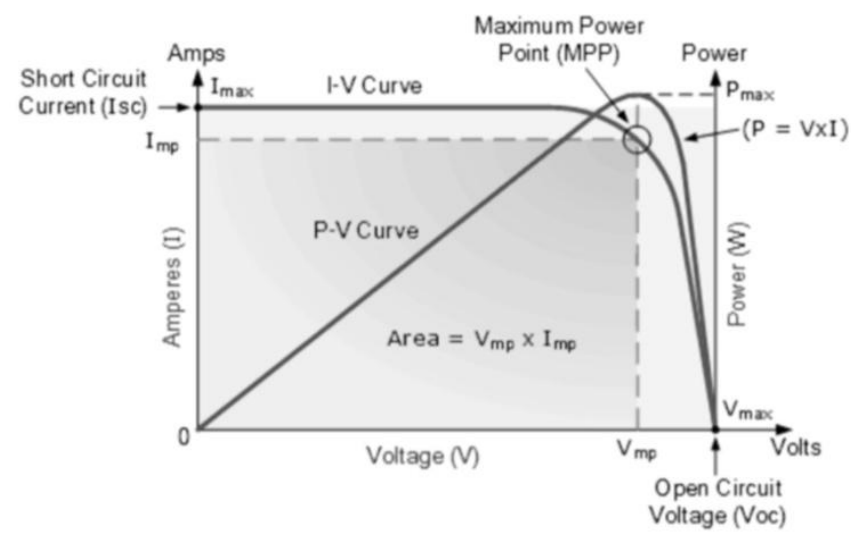

Fig. 2. Photovoltaic I-V characteristics.

To get the gradient of the output power vary the operating current until the gradient reaches almost zero. This method refers the actual output power detection of the PV and thus the Maximum Power Point Tracking can be obtained even if the optimum operating points fluctuate due to irradiation and temperature problems [3]. The algorithm flowchart is as shown in Figure 3.

Small PV panels that are put together make Photovoltaic arrays. Then the current-voltage curve of a Photovoltaic array will be as shown in Figure 4. Connect PV modules in series combination to boost voltage or in parallel to boost current. The electrical power generated by these combinations will still be the product of the voltage and the current. Whatever the combination maybe, MPP always stays at the top right corner.

Variable resistors like Light Dependent Resistor (LDR) adapt to different lighting conditions and brightness which in turn helps in calibrating Photosensors sensibility. It is possible to determine the area of maximum light intensity by photosensors as they acquire analog signals since they contain the signal conditioner integrated circuits and amplifier.

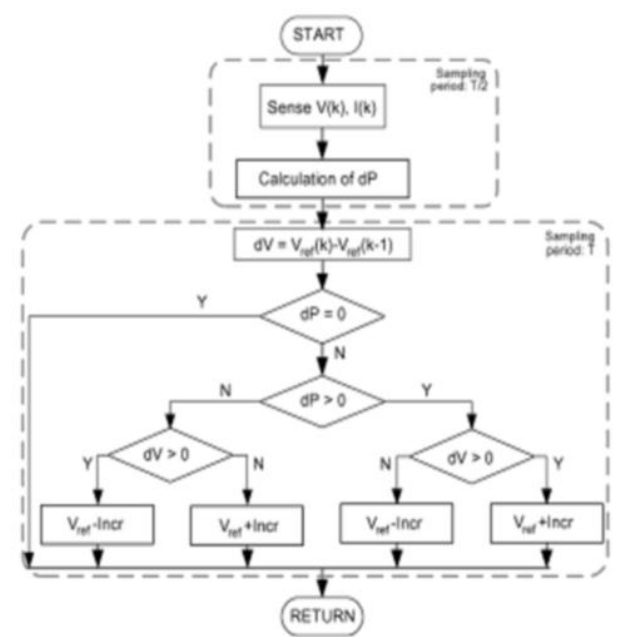

Fig. 3. Algorithm flowchart [3]. 


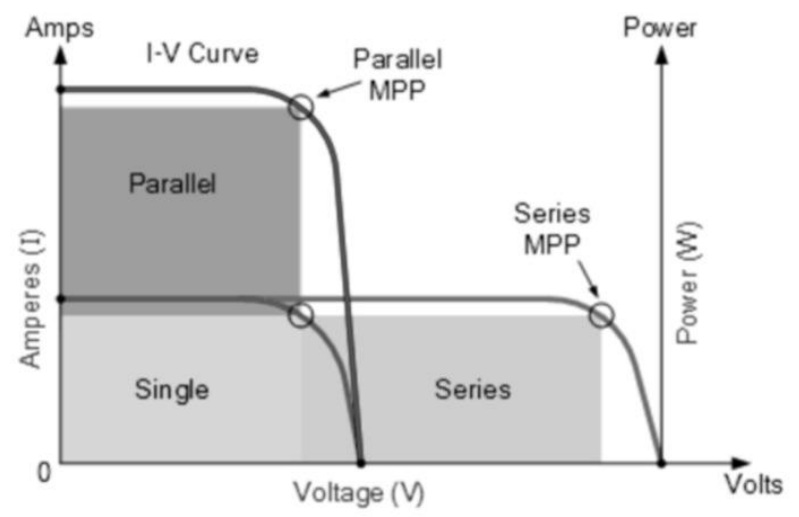

Fig. 4. I-V Curve of a PV array.

Light intensity values that are analogous are compared in pairs and the difference is used to control the signal for elevation and azimuth required by the solar tracking system. Signal duty cycle determines the servo motors required rotation which is controlled by pulse width modulation.

Power source: Pulse width modulation (PWM) is used to control the battery charging system. When the signal is applied to one of the charger system terminals it supplies power to each battery according to a programmed algorithm.

Capacitor and a current/voltage (I/V) sensor are placed between the automatic solar tracking system and battery charger system. The use of a capacitor is needed when power generated from solar is not enough to provide appropriate voltage and it helps to hold the power at the battery charger input to prevent the voltage from falling below the charging voltage of the battery. To detect the current and voltage levels of the charger module provided by the solar panels, I/V sensor is used. The controller consists of an algorithm which according to Maximum Power Point Tracking (MPPT) regulates the charge by increasing the charger modules output current. The monitoring system helps in maximizing the overall lifetime and energy of the battery. The incorporated module consists of two selectors. Each selector is connected to batteries in parallel. The measurement of the capacity of the battery, the one in charge and other bring discharged is continuously measured by the battery charger system [6].

\section{MACHINE VISION BASED ROW GUIDANCE SYSTEM}

One of the best methods for navigation is machine visionbased row guidance system. This is achieved by vision (with yaw and pitch motions) and ultrasonic sensors, which are widely available and are affordable. They capture the images and processes them with image processing algorithms with morphological features to get cartesian coordinates and edges that are given to the Raspberry Pi. This traversal path derived also guides the bot through corners. Sequence of operations are performed to get these coordinates: 1. Acquisition of Image, 2. Removal of noise, 3. Sobel operation, 4. Conversion from Red, Green, Blue (RGB) color space to grayscale, 5. Extraction of the edges,

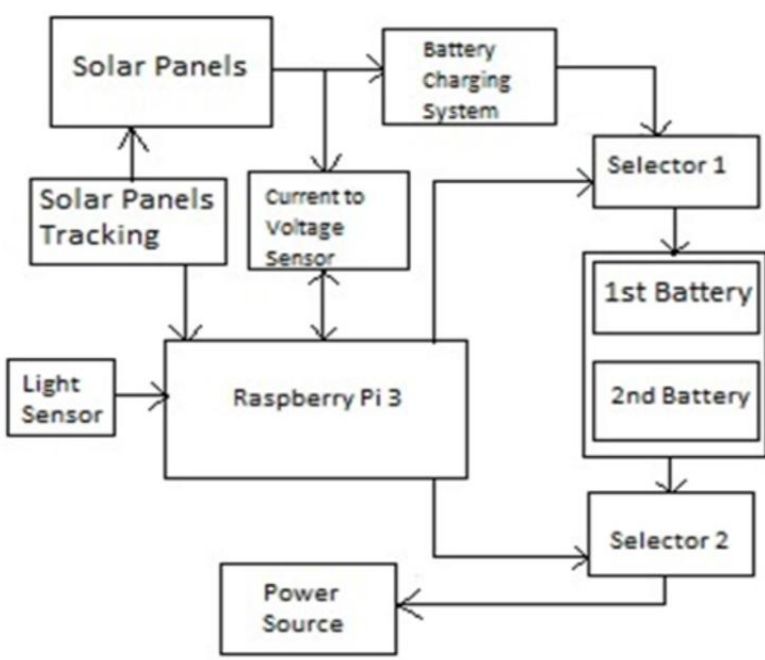

Fig. 5. Solar tracking with battery management.

6. Calculation of the centroid, and 7. Conversion of this centroid to cartesian coordinates.

Once the map is decided, ultra-sonic sensors are used to check for obstacles, if none are detected, the bot moves further according to map. To obtain self-localization the bot uses Global Positioning System (GPS). Algorithm used cross correlates these GPS coordinates to that of the map coordinates to move in accordance with the map. This happens continuously till the end point is reached [2].

\section{CONCLUSION}

The proposed paper presents an automated bot with multiple capabilities that can carry out multiple operations on an agricultural field. Methods proposed in this paper defines the use of solar panel tracking i.e., movable photovoltaic panels for maximum power utilization. The machine vision technology in the bot helps it to overcome the challenges faced in the navigation on the agricultural field. The battery charging and switching mechanism boost the ability of the bot to preserve and use the power more efficiently. With all these capabilities combined in a bot, we can rationalize our proposed model to be effective, eco-friendly and utilizable by the agricultural industry.

\section{REFERENCES}

1. Palepu V Santhi, Nellore Kapileswar, Vijay K. R. Chenchelaand Venkata Siva Prasad, "Sensor and Vision based Autonomous AGRIBOT for Sowing seeds," International Conference on Energy, Communication, Data Analytics and Soft Computing (ICECDS), 2017.

2. Snehal S Warekar and B. T. Salokhe, "Solar powered robotic vehicle," International Journal of Advanced Research in Computer and Communication Engineering Vol. 4, Issue 5, May 2015.

3. R. C. Prasad, "Design and Implementation of MPPT Algorithm for Solar Energy System," International Journal of Advanced Research in Computer Ccience and Software Engineering, Vol. 3, Issue 10, October 2013

4. Theodore Amissah Ocran, Cao Juny, Cao Binggang, and Sun Xinghua, "Artificial Neural Network Maximum Power Point Tracker for Solar Electric Vehicle," Tsinghua Science and Technology, Vol. 10, No. 2, pp. 204-208, April 2005. 
5. Tom as de J. Mateo Sanguino and Justo E. Gonz alez Ramos, "Smart Host Micro controller for Optimal Battery Charging in a Solar-Powered Robotic Vehicle," IEEE/ASME Transactions on Mechatronics, vol. 18, no. 3, June 2013.

6. N Kemal Ure, Girish Chowdhary, Tuna Toksoz, Jonathan P How, Matthew A. Vavrina, and John Vian, "An Automated Battery Management System to Enable Persistent Missions With Multiple Aerial Vehicles," IEEE/ASME Transactions on Mechatronics, 2013.

\section{AUTHORS PROFILE}

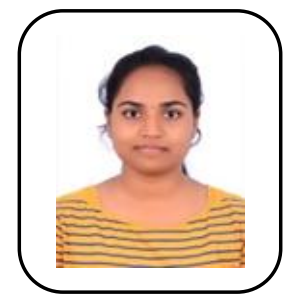

Gopireddy Sirisha is a Final year B.Tech (Electronics and Communication) student of VIT Chennai. She is currently serving as a teaching assistant for the course Signals and Systems. Her major research domains are Embedded Systems, Embedded Security and Internet-of-Things. She is highly motivated towards developing secure Embedded Systems for solving real world problems.

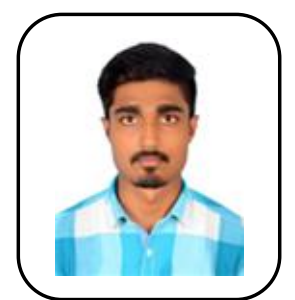

Harshdeepsinh Parmar is a Final year B.Tech (Electronics and Communication) student of VIT Chennai. He graduated his Higher Secondary from P. P. Savani Chaitanya Vidhya Sankul affiliated to CBSE. He served as a teaching assistant for the course Embedded System Design with profound knowledge in Programming in $\mathrm{C}$, $\mathrm{C}++$ and Embedded Systems.

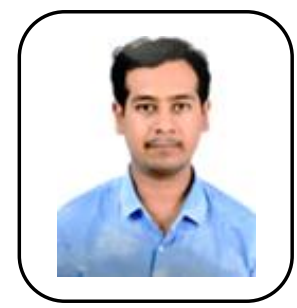

Konduri Subrahmanya Hemanth is a Final year B.Tech (Electronics and Communication) student of VIT Chennai. His areas of research are Embedded Systems, Internet of Things and Wireless Networking. He is interested in Industry Control and Automation using Wireless Sensor Networks.

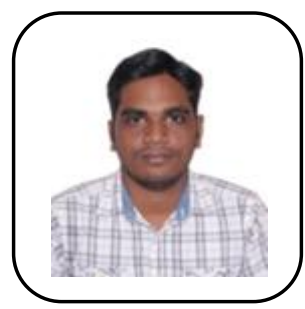

Sathiya Narayanan is currently working as an Assistant Professor in Vellore Institute of Technology, Chennai. He has more than eight years of academic research experience. He received his B.E. degree from Anna University, Chennai - India, and his M.S. and Ph.D. degrees from Nanyang Technological University (NTU), Singapore. His areas of expertise are image/video processing, compressive imaging and signal processing for communication. He was the recipient of the NTU Research Student Scholarship 2011-2015 and NTU-EEE Outstanding Teaching Assistant Award 2014. From October 2015 to April 2018, he served as a Research Associate/Fellow in NTU. 